\title{
Genome wide analysis of quantitative disease resistance against Verticillium wilt in the model legume Medicago truncatula
}

\author{
M. Mazurier ${ }^{1}$, M. Toueni ${ }^{1}$, A. Sbeiti ${ }^{1}$, M.C. Tardin' ${ }^{2}$, M.C. Gras ${ }^{2}$, M. Rickauer ${ }^{1}$, \\ L. Gentzbitte, C. Ben ${ }^{1 *}$ \\ ${ }^{1}$ EcoLab, Université de Toulouse, CNRS, Toulouse INP, UPS, Toulouse, France \\ ${ }^{2} R 2 n$ - Centre de Recherche Le Bourg, Druelle, France \\ *e-mail:ben@ensat.fr
}

Key words: GWAS, quantitative trait loci (QTL), candidate gene, root disease, biodiversity, functional validation, biotechnology

Motivation and Aim: Legumes are one of the most important crop families with high nutritional value thanks to their nitrogen-fixing symbiosis with rhizobia. However, they are prone to many diseases which reduce yields. The interaction between the model legume Medicago truncatula and the root pathogen Verticillium alfalfae is studied to investigate the genetic mechanisms involved in quantitative disease resistance against Verticillium wilt in Legumes.

Methods and Algorithms: Genome-wide association studies (GWAS) were conducted on disease parameters assessing disease development and plant colonization in a collection of $242 \mathrm{M}$. truncatula accessions. The disease parameters were obtained after fitting nonlinear mixed models to the time-course disease symptoms. The analyses on adjusted means from an augmented-block experimental design were performed with the TASSEL v5 software by implementing a linear mixed model including kinship as the variancecovariance matrix of the random genetic effects, and population structure as fixed effects (EMMAX algorithm). A set of 5M SNPs was used.

Results: Symptom scoring and fungus reisolation in 242 M. truncatula ecotypes highlighted a large biodiversity of the response to $V$. alfalfae ( $\mathrm{Va}$ V31-2 strain) with a continuous range from fully resistant to susceptible lines. The genome wide association study (GWAS) on various modeled disease parameters pinpointed quantitative trait loci (QTL) on chromosome 1,7 and 8. Both phenotypic and genetic analyses thus suggest the occurrence of different resistance mechanisms in $M$. truncatula populations towards $V$. alfalfae. Among five candidate genes localized under a previously described major QTL for $V$. alfalfae resistance on chromosome 7 [1], only two genes, encoding for proteins involved in ubiquitination and lipid metabolism, were found to be expressed in response to $V$. alfalfae. The latter one was validated as a disease susceptibility gene towards $\mathrm{Va}$ V31.2 in M. truncatula. Gene silencing using artificial microRNA in A17 (resistant) and F83005.5 (susceptible) lines decreases $V a$ V31-2 colonization rate on transgenic roots whereas overexpression of the gene increases the colonization rate in A17.

Conclusion: Understanding Verticillium resistance and its genetic control in the model legume M. truncatula will help to develop breeding strategies for legume plants which are important components of sustainable agriculture and ecosystems.

Acknowledgements: M. Mazurier was supported by a PhD scholarship from the French "Ministère de la Recherche et de l'Enseignement Supérieur», M. Toueni by a doctoral grant from Mayotte department, and A. Sbeiti by a PhD grant from "Association des Jeunes de KFARSIR", managed by Toufik Sbeiti et Mariam Malek, Lebanon. We thank J.M. Prosperi for providing seeds and maintaining a large part of the Medicago truncatula collections. We thank the Medicago Hapmap international consortium, mainly funded by the Plant Genome Program of the National Science Foundation (USA), for providing M. truncatula genome resequencing data.

\section{References}

1. Ben C. et al. (2013) Natural diversity in the model legume Medicago truncatula allows identifying distinct genetic mechanisms conferring partial resistance to Verticillium wilt. J Exp Bot. 64(1):317-332. 